\title{
ON ATTRACTIVITY FOR NONAUTONOMOUS SYSTEMS
}

\author{
By \\ XINZHI LIU \\ University of Waterloo, Waterloo, Ontario, Canada
}

1. Introduction. The original Lyapunov theorems have been extended, generalized, and weakened in various aspects. It is well known that Barbashin and Krasovskii [1] generalized Lyapunov's theorem on asymptotic stability to the case when the time derivative of a Lyapunov function is only semi-negative definite for periodic differential equations. Inspired by their results, LaSalle considered autonomous systems and made a number of contributions [7]. The general result is now known as LaSalle's invariance principle, which is recognized to be one of the most useful results in applications. For example, it allows using the total energy as a Lyapunov function to obtain asymptotic stability in mechanical problems with dissipation. The key idea of the invariance principle is to use Lyapunov's method to locate an attractive set $E$ and then to refine the result by using invariance properties of a subset of $E$. For nonautonomous systems, Yoshizawa [12] gave a sufficient condition for the attractivity of a closed set. Later, his result was improved by Burton [2] and Haddock [3]. See also LaSalle [8]. Further improvement was given by Hatvani [4]. However, the invariance principle fails to apply to nonautonomous systems since one cannot rely, in general, on any invariance property of the limit sets. For example, consider the linear oscillator with time-varying friction

$$
x^{\prime \prime}+\left(e^{t}+2\right) x^{\prime}+x=0
$$

or equivalently the system

$$
\left\{\begin{array}{l}
x^{\prime}=y \\
y^{\prime}=-\left(e^{t}+2\right) y-x .
\end{array}\right.
$$

Let $V(x, y)=\frac{1}{2}\left(x^{2}+y^{2}\right)$. Then the time derivative of $V$ along the solutions of Eq. (1.2) is $D^{+} V(x, y)=-\left(e^{t}+2\right) y^{2}$. Thus the set $E$ is the whole $x$-axis, and it can be shown that $E$ attracts all the solutions and contains no complete positive semitrajectories except $(0,0)$. However, the null solution of Eq. (1.2) is not asymptotically stable. Thus the attractiveness of a single set is often insufficient to give the desired result. Matrosov [10] observed this and using an additional Lyapunov-like function with suitable properties in the vicinity of the set $E$ where $D^{+} V(t, x) \equiv 0$

Received July 2, 1991.

1991 Mathematics Subject Classification. Primary 34D20, 34D45, 93D30.

Research partially supported by NSERC Canada.

(C)1993 Brown University 
proved asymptotic stability. Improvement has been made recently by Salvadori [11], the author [9], and Hatvani [5]. In this paper, we attempt to give a new approach, i.e., when a larger attractive set containing the desired invariant set is found, it is advantageous, instead of imposing compensating conditions on this set, to choose another convenient set relative to which we may impose suitable assumptions to yield attractivity of the desired invariant set. In case the first refinement is unsatisfactory, this refining process can be continued until the desired result is obtained.

2. Preliminaries. Consider the differential system

$$
x^{\prime}=f(t, x), \quad x\left(t_{0}\right)=x_{0}, \quad t_{0} \geq 0,
$$

where $f \in C\left[R_{+} \times R^{n}, R^{n}\right]$. Let $x(t)=x\left(t, t_{0}, x_{0}\right)$ be any solution of Eq. (2.1) existing on $\left[t_{0}, \alpha\right)$. For simplicity, we always assume $\alpha=+\infty$. For $x \in R^{n}$, $E \subset R^{n}$, we define the distance between $x$ and $E$ by $d(x, E)=\inf \{|x-y| ; y \in E\}$, $|\cdot|$ being any convenient norm in $R^{n}$. For $\delta>0, S(E, \delta)=\left\{x \in R^{n}, d(x, E)<\delta\right\}$ and $S^{c}(E, \delta)=R^{n} \backslash S(E, \delta)$. We denote by $[a]_{+},[a]_{-}$the positive and negative part of a real number $a$, respectively, i.e., $[a]_{+}=\max [0, a],[a]_{-}=\max [0,-a]$.

Definition 2.1. A measurable function $\lambda: R_{+} \rightarrow R_{+}$is said to be integrally positive if

$$
\int_{I} \lambda(s) d s=\infty
$$

for every set $I=\bigcup_{k=1}^{\infty}\left[\alpha_{k}, \beta_{k}\right], \beta_{k}<\alpha_{k+1}$, and $\beta_{k}-\alpha_{k} \geq \delta>0$.

Definition 2.2. Let $\Omega \subset R^{n}$ be an open set. A function $W: R_{+} \times \Omega \rightarrow R_{+}$is said to be positive definite with respect to a closed set $E \subset \Omega$ if $W(t, x) \equiv 0$ for $x \in E$ and for any $\varepsilon>0$ there exists $\delta(\varepsilon)>0$ such that

$$
W(t, x) \geq \delta(\varepsilon) \quad \text { for } x \in S^{c}(E, \varepsilon) \cap \Omega \text { and } t \in R_{+} .
$$

Definition 2.3. A function $W: R_{+} \times \Omega \rightarrow R_{+}$is said to be weakly decrescent with respect to a closed set $E \subset \Omega$ if for any $\varepsilon>0$ there exists $\delta=\delta(t, \varepsilon)>0$ such that

$$
W(t, x)<\varepsilon, \quad x \in S(E, \delta) \cap \Omega .
$$

$W(t, x)$ is decrescent if the above $\delta$ is independent of $t$.

Definition 2.4. Let $M \subset R^{n}$ be a closed set and $x(t)=x\left(t, t_{0}, x_{0}\right)$ be any solution of Eq. (2.1). Then the set $M$ is said to be

$\left(\mathrm{S}_{1}\right)$ stable if for every $\varepsilon>0$ and $t_{0} \geq 0$ there exists $\delta=\delta\left(t_{0}, \varepsilon\right)>0$ such that $d\left(x_{0}, M\right)<\delta$ implies $d(x(t), M)<\varepsilon, t \geq t_{0}$;

$\left(\mathrm{S}_{2}\right)$ uniformly stable if $\left(\mathrm{S}_{1}\right)$ holds with $\delta$ being independent of $t_{0}$;

$\left(\mathrm{S}_{3}\right)$ attractive if for each $t_{0} \geq 0$ there exists $\delta=\delta\left(t_{0}\right)>0$ such that $d\left(x_{0}, M\right)<$ $\delta$ implies $d(x(t), M) \rightarrow 0$ as $t \rightarrow \infty$;

$\left(\mathrm{S}_{4}\right)$ uniformly attractive if for some $\delta>0$ and any $\varepsilon>0$ there exists $T=$ $T(\delta, \varepsilon)>0$ such that for $t_{0} \geq 0, d\left(x_{0}, M\right)<\delta$ implies $d(x(t), M)<\varepsilon$, $t \geq t_{0}+T$

$\left(\mathrm{S}_{5}\right)$ asymptotically stable if $\left(\mathrm{S}_{1}\right)$ and $\left(\mathrm{S}_{3}\right)$ hold simultaneously;

$\left(\mathrm{S}_{6}\right)$ uniformly asymptotically stable if $\left(\mathrm{S}_{2}\right)$ and $\left(\mathrm{S}_{4}\right)$ hold together. 
Definition 2.5. Let $V \in C\left[R_{+} \times R^{n}, R\right]$; the upper right derivative of $V(t, x)$ with respect to the differential system $(2.1)$ is defined as

$$
D^{+} V(t, x)=\limsup _{h \rightarrow 0^{+}} \frac{1}{h}[V(t+h, x+h f(t, x))-V(t, x)] .
$$

It is well known that if $V(t, x)$ is locally Lipschitz in $x$ then for any solution $x(t)=x\left(t, t_{0}, x_{0}\right)$ of Eqs. (2.1) we have

$$
\limsup _{h \rightarrow 0^{+}} \frac{1}{h}[V(t+h, x(t+h))-V(t, x(t))]=D^{+} V(t, x(t)) .
$$

We denote by $K$ the class of continuous functions $\sigma: R_{+} \rightarrow R_{+}$, which are strictly increasing and vanishing at zero.

3. Main results. We begin by proving a theorem that provides a new approach in the study of asymptotic behaviours of solutions of nonautonomous systems of differential equations.

Theorem 3.1. Assume that there exist functions $V \in C\left[R_{+} \times \Omega, R\right], \Omega$ being an open subset of $R^{n}$, and $W_{1}, W_{2} \in C\left[R_{+} \times \Omega, R_{+}\right] ; V(t, x), W_{1}(t, x)$, and $W_{2}(t, x)$ are locally Lipschitz in $x . V(t, x)$ is bounded from below, and for each $i=1,2, W_{i}(t, x)$ is positive definite with respect to a closed set $E_{i} \subset \Omega, E_{1} \cap E_{2} \neq$ $\varnothing$. Suppose further that

(i) $D^{+} V(t, x) \leq-\lambda(t) c_{1}\left(W_{1}(t, x)\right)+e_{1}(t),(t, x) \in R \times \Omega$, where $\lambda(t)$ is integrally positive, $c_{1} \in K$, and $e_{1}: R_{+} \rightarrow R_{+}$is integrable over $R_{+}$;

(ii) for any solution $x(t)$ of Eqs. (2.1), the function $\int_{t_{0}}^{t}\left[D^{+} W_{1}(s, x(s))\right]_{ \pm} d s$ is uniformly continuous on $R_{+}$;

(iii) $D^{+} W_{2}(t, x) \leq-c_{2}\left(W_{2}(t, x)\right)+\psi\left(W_{1}(t, x)\right)+e_{2}(t),(t, x) \in R_{+} \times \Omega$, where $c_{2} \in K, \psi: R_{+} \rightarrow R_{+}$is continuous and $\psi(0)=0$, and $e_{2}: R_{+} \rightarrow R_{+}$is integrable over $R_{+}$.

Then $x(t) \rightarrow E_{1} \cap E_{2}$ as $t \rightarrow \infty$ for any solution $x(t)=x\left(t, t_{0}, x_{0}\right)$ of Eq. (2.1), which remains in $\Omega$ in the future.

Proof. Let $x(t)=x\left(t, t_{0}, x_{0}\right)$ be a solution of Eqs. (2.1) existing on $\left[t_{0}, \infty\right)$ and set $v(t)=V(t, x(t)), w_{1}(t)=W_{1}(t, x(t))$, and $w_{2}(t)=W_{2}(t, x(t))$. Then from condition (i) we have

$$
D^{+} v(t) \leq-\lambda(t) c_{1}\left(w_{1}(t)\right)+e_{1}(t), \quad t \geq t_{0} .
$$

We are going to show $\lim _{t \rightarrow \infty} w_{1}(t)=0$. Clearly, $\liminf _{t \rightarrow \infty} w_{1}(t)=0$. Suppose that $\lim _{t \rightarrow \infty} w_{1}(t) \neq 0$. For definiteness, we assume that $[\cdot]_{+}$stands in (ii). Then for some $\gamma>0$, we can choose a sequence

$$
t_{0}<\alpha_{1}<\beta_{1}<\cdots<\alpha_{i}<\beta_{i}<\cdots
$$

such that, for $i=1,2, \ldots$,

$$
w_{1}\left(\alpha_{i}\right)=\gamma, \quad w_{1}\left(\beta_{i}\right)=2 \gamma, \quad \gamma \leq w_{1}(t) \leq 2 \gamma, \quad t \in\left[\alpha_{i}, \beta_{i}\right] .
$$

Thus we have

$$
0<\gamma=w_{1}\left(\beta_{i}\right)-w_{1}\left(\alpha_{i}\right) \leq \int_{\alpha_{i}}^{\beta_{i}}\left[D^{+} W_{1}(s, x(s))\right]_{+} d s .
$$


It then follows from condition (ii) that there exists a positive constant $\delta>0$ such that

$$
\beta_{i}-\alpha_{i} \geq \delta, \quad i=1,2, \ldots
$$

Let $I=\bigcup_{i=1}^{\infty}\left[\alpha_{i}, \beta_{i}\right]$. Then by Eqs. (3.1)-(3.3) we have

$$
\lim _{t \rightarrow \infty} v(t) \leq v\left(t_{0}\right)-c_{1}(\gamma) \int_{I} \lambda(s) d s+\int_{t_{0}}^{\infty} e_{1}(s) d s=-\infty,
$$

which is a contradiction.

Thus we have proved $\lim _{t \rightarrow \infty} w_{1}(t)=0$. Since $W_{1}(t, x)$ is positive definite with respect to $E_{1}$, it follows that $x(t) \rightarrow E_{1}$ as $t \rightarrow \infty$.

By condition (iii), we have

$$
D^{+} w_{2}(t) \leq-c_{2}\left(w_{2}(t)\right)+\psi\left(w_{1}(t)\right)+e_{2}(t), \quad t \geq t_{0} .
$$

We claim that $\liminf _{t \rightarrow \infty} w_{2}(t)=0$. If this is false, then there exist constants $d$, $T_{1}>0$ such that

$$
w_{2}(t) \geq d \quad \text { for } t \geq t_{0}+T_{1} .
$$

Since $\lim _{t \rightarrow \infty} w_{1}(t)=0$ and $\psi(0)=0$, there exists $T_{2}>0$ such that

$$
\psi\left(w_{1}(t)\right) \geq \frac{c_{2}(d)}{2} \text { for } t \geq t_{0}+T_{2} .
$$

Choose $T=\max \left\{T_{1}, T_{2}\right\}$ so that, for $t \geq t_{0}+T$, by Eqs. (3.4)-(3.6),

$$
D^{+} w_{2}(t) \leq-\frac{c_{2}(d)}{2}+e_{2}(t), \quad t \geq t_{0}+T .
$$

Integrating Eq. (3.7), we get

$$
w_{2}(t) \leq w_{2}\left(t_{0}+T\right)-\frac{c_{2}(d)}{2}\left(t-T-t_{0}\right)+\int_{t_{0}+T}^{t} e_{2}(s) d s, \quad t \geq t_{0}+T,
$$

and this implies that $\limsup _{t \rightarrow \infty} w_{2}(t)=-\infty$, which is absurd. Suppose that $\lim \sup _{t \rightarrow \infty} w_{2}(t) \neq 0$. Then we can find a sequence

$$
t_{0}<t_{1}^{(1)}<t_{1}^{(2)}<\cdots<t_{i}^{(1)}<t_{i}^{(2)}<\cdots, \quad t_{i}^{(1)} \rightarrow \infty \text { as } i \rightarrow \infty
$$

such that

$$
w_{2}\left(t_{i}^{(1)}\right)=\sigma, \quad w_{2}\left(t_{i}^{(2)}\right)=2 \sigma, \quad \sigma \leq w_{2}(t) \leq 2 \sigma, \quad t \in\left[t_{i}^{(1)}, t_{i}^{(2)}\right],
$$

for some constant $\sigma>0$.

Since $\lim _{t \rightarrow \infty} w_{1}(t)=0$, there exists $T_{3}>0$ such that

$$
\psi\left(w_{1}(t)\right) \leq \frac{c_{2}(\sigma)}{3}, \quad t \geq t_{0}+T_{3} .
$$

Also $e_{2}(t)$ integrable over $R_{+}$implies that for some $T_{4}>0$,

$$
e_{2}(t) \leq \frac{c_{2}(\sigma)}{3}, \quad t \geq t_{0}+T_{4} .
$$

It thus follows from Eqs. (3.4), (3.8)-(3.10) that for sufficiently large $i$,

$$
D^{+} w_{2}(t) \leq-\frac{c_{2}(\sigma)}{3}, \quad t \in\left[t_{i}^{(1)}, t_{i}^{(2)}\right]
$$


which implies that $w_{2}(t)$ is decreasing on $\left[t_{i}^{(1)}, t_{i}^{(2)}\right]$. Hence, $w_{2}\left(t_{i}^{(2)}\right)<w_{1}\left(t_{i}^{(1)}\right)$, which is a contradiction to Eqs. (3.8). Thus $\lim _{t \rightarrow \infty} w_{2}(t)=0$. Since $w_{2}(t, x)$ is positive definite with respect to $E_{2}, x(t) \rightarrow E_{2}$ as $t \rightarrow \infty$. This, together with the conclusion $x(t) \rightarrow E_{1}$ as $t \rightarrow \infty$, implies $x(t) \rightarrow E_{1} \cap E_{2}$ as $t \rightarrow \infty$, completing the proof.

REMARK 1. Condition (ii) is satisfied if

$$
-p(t) \leq D^{+} W_{1}(t, x) \quad \text { or } \quad D^{+} W_{1}(t, x) \leq q(t), \quad(t, x) \in R_{+} \times \Omega,
$$

where $p, q: R_{+} \rightarrow R_{+}$are measurable functions such that $\int_{0}^{t} p(s) d s$ and $\int_{0}^{t} q(s) d s$ are uniformly continuous on $R_{+}$.

REMARK 2. If $e_{1}(t) \equiv e_{2}(t) \equiv 0, E=E_{1} \cap E_{2}$ is an invariant set of Eqs. (2.1), and $V(t, x)$ is positive definite with respect to $E$, then $V(t, x)$ is weakly decrescent with respect to $E$ implies that $E$ is asymptotically stable.

EXAMPLE 3.1. Consider the nonlinear system

$$
\left\{\begin{array}{l}
x^{\prime}=y, \\
y^{\prime}=-\cos ^{2} t\left(1+x^{2}+y^{2}\right) y^{3}-x^{3}+b e^{-t} \sin \left(1+x^{2}\right) /\left(1+x^{2}+y^{2}\right) .
\end{array}\right.
$$

Let $U(t, x, y)=e^{\left(e^{t}-1\right)}\left[\frac{1}{2} x^{4}+\frac{1}{2} y^{2}+|b|\right]$. Then we have $D^{+} U(t, x, y) \leq 0$, which implies that all solutions of Eqs. (3.11) are uniformly bounded and defined for all $t \geq t_{0}$. Choose $M>0$ such that

$$
\sqrt{x^{2}(t)+y^{2}(t)} \leq M, \quad t \geq t_{0},
$$

for all solutions $(x(t), y(t))$ of Eqs. (3.11). Let $V(x, y)=\frac{1}{4} x^{4}+\frac{1}{2} y^{2}$. Then the time derivative of $V(x, y)$ along Eqs. (3.11) is

$$
D^{+} V(x, y) \leq-\cos ^{2} t y^{4}+M e^{-t} .
$$

Clearly, $\cos ^{2} t$ is integrally positive and $M e^{-t}$ is integrable over $R_{+}$. Set $W_{1}(x, y)=$ $y^{2}$. Then

$$
D^{+} W_{1}(x, y) \leq M^{4}+|b| .
$$

Let $W_{2}(x, y)=\frac{1}{2}(x+y)^{2}$. Then it is easy to obtain

$$
D^{+} W_{2}(x, y) \leq-2 M^{2} W_{2}(x, y)+\psi\left(W_{1}(x, y)\right)+2 M e^{-t},
$$

where $\psi(u)=\left(3 M^{3}+M\right) \sqrt{u}+\left(M^{2}+1\right) u+M u^{3 / 2}, \psi(u)$ is continuous on $R_{+}$, and $\psi(0)=0$. Thus all conditions of Theorem 3.1 are satisfied with $E_{1}$ being the line $y=0$ and $E_{2}$ the line $x+y=0$. Therefore all solutions of Eqs. (3.11) approach $(0,0)$ as $t \rightarrow \infty$.

If $b=0$, then it is easy to see that the system (3.11) admits a trivial solution, which is globally asymptotically stable.

It should be noted that in order to drive the solutions of Eqs. (2.1) into the desired invariant set, we employed in Theorem 3.1 a second Lyapunov-like function $W_{2}$ related to another set $E_{2}$ and proved that all solutions of Eqs. (2.1) starting in $E_{1}$, the initially obtained attractive set, would tend to $E_{2}$. The present approach seems 
more natural and transparent, and it actually provides us with a refining procedure. In case the first refinement is unsatisfactory, this refining process can be continued until the desired result is obtained. The following result is a natural generalization of Theorem 3.1.

TheOREM 3.2. Assume that there exist functions $V \in C\left[R_{+} \times \Omega, R\right], W_{1}, W_{2}, \ldots$, $W_{N} \in C\left[R_{+} \times \Omega, R_{+}\right]$such that $V(t, x), W_{1}(t, x), \ldots, W_{N}(t, x)$ are locally Lipschitz in $x, V(t, x)$ is bounded from below, and for each $i=1,2, \ldots, N, W_{i}(t, x)$ is positive definite with respect to $E_{i} \subset \Omega$, where $\bigcap_{i=1}^{N} E_{i} \neq \varnothing$. Suppose further that

(i) $D^{+} V(t, x) \leq-\lambda(t) c_{11}\left(W_{1}(t, x)\right)+e_{1}(t),(t, x) \in R_{+} \times \Omega$, where $c_{11} \in K, \lambda(t)$ is integrally positive, and $e_{1}: R_{+} \rightarrow R_{+}$is integrable over $R_{+}$;

(ii) for any solution $x(t)$ of Eqs. (2.1) the function $\int_{t_{0}}^{t}\left[D^{+} W_{1}(s, x(s))\right]_{ \pm} d s$ is uniformly continuous on $R_{+}$;

(iii)

$$
\begin{aligned}
D^{+} W_{2}(t, x) \leq & -c_{22}\left(W_{2}(t, x)\right)+\psi_{21}\left(W_{1}(t, x)\right)+e_{2}(t), \quad(t, x) \in R_{+} \times \Omega, \\
D^{+} W_{3}(t, x) \leq & -c_{33}\left(W_{3}(t, x)\right)+\psi_{32}\left(W_{2}(t, x)\right) \\
& +\psi_{31}\left(W_{1}(t, x)\right)+e_{3}(t), \quad(t, x) \in R_{+} \times \Omega, \\
\cdots & \\
D^{+} W_{N}(t, x) \leq & -c_{N N}\left(W_{N}(t, x)\right)+\psi_{N N-1}\left(W_{N-1}(t, x)\right)+\cdots \\
& +\psi_{N 1}\left(W_{1}(t, x)\right)+e_{N}(t),(t, x) \in R_{+} \times \Omega,
\end{aligned}
$$

where for each $i=2, \ldots, N, c_{i i} \in K$, and $e_{i}: R_{+} \rightarrow R_{+}$is integrable over $R_{+}$, and $\psi_{21}, \psi_{31}, \psi_{32}, \ldots, \psi_{N 1}, \ldots, \psi_{N N-1}: R_{+} \rightarrow R_{+}$are continuous and vanishing at zero. Then every solution $x(t)=x\left(t, t_{0}, x_{0}\right)$ of Eqs. (2.1), which remains in $\Omega$ in the future, approaches the set $\bigcap_{i=1}^{N} E_{i}$ as $t$ tends to infinity.

Proof. It follows from Theorem 3.1 that for any solution $x(t)=x\left(t, t_{0}, x_{0}\right)$ of (2.1) with $x(t) \in \Omega, t \geq t_{0}$,

$$
\lim _{t \rightarrow \infty} W_{1}(t, x(t))=\lim _{t \rightarrow \infty} W_{2}(t, x(t))=0 .
$$

Suppose that for $k \geq 2$,

$$
\lim _{t \rightarrow \infty} W_{i}(t, x(t))=0, \quad i=1,2, \ldots, k-1 .
$$

We are going to show that $\lim _{t \rightarrow \infty} W_{k}(t, x(t))=0$. We first claim that

$$
\liminf _{t \rightarrow \infty} W_{k}(t, x(t))=0 \text {. }
$$

If this is not true, then there exist constants $d, T_{k}>0$ such that

$$
W_{k}(t, x(t)) \geq d, \quad t \geq t_{0}+T_{k} .
$$

By Eq. (3.12) we see that for each $i=1,2, \ldots, k-1$, there is a $T_{i}>0$ such that

$$
\psi_{k i}\left(W_{i}(t, x(t))\right) \leq \frac{c_{k k}(d)}{k}, \quad t \geq t_{0}+T_{i} .
$$


From condition (iii), we obtain

$$
\begin{aligned}
D^{+} W_{k}(t, x(t)) \leq & -c_{k k}\left(W_{k}(t, x(t))+\psi_{k k-1}\left(W_{k-1}(t, x(t))\right)+\cdots\right. \\
& +\psi_{k 1}\left(W_{1}(t, x(t))\right)+e_{k}(t), \quad t \geq t_{0} .
\end{aligned}
$$

Integration of the inequality (3.15) yields

$$
W_{k}(t, x(t)) \leq W_{k}\left(t_{0}+T\right)-\frac{c_{k k}(d)}{k}\left(t-T-t_{0}\right)+\int_{t_{0}+T}^{t} e_{k}(s) d s, \quad t \geq t_{0}+T,
$$

where $T=\max \left\{T_{1}, T_{2}, \ldots, T_{k}\right\}$. This implies that $\limsup _{t \rightarrow \infty} W_{k}(t, x(t))=-\infty$, which is absurd. If $\limsup _{t \rightarrow \infty} W_{k}(t, x(t)) \neq 0$, then there exists a constant $\sigma>0$ and a sequence

$$
t_{0}<\alpha_{1}<\beta_{1}<\cdots<\alpha_{i}<\beta_{i}<\cdots, \quad \alpha_{i} \rightarrow \infty \text { as } i \rightarrow \infty
$$

such that

$$
\begin{gathered}
W_{k}\left(\alpha_{i}, x\left(\alpha_{i}\right)\right)=\sigma, \quad W_{k}\left(\beta_{i}, x\left(\beta_{i}\right)\right)=2 \sigma, \\
\sigma \leq W_{k}(t, x(t)) \leq 2 \sigma, \quad t \in\left[\alpha_{i}, \beta_{i}\right] .
\end{gathered}
$$

Again by Eqs. (3.12), there exists, for each $i=1,2, \ldots, k-1$, a constant $\widetilde{T}_{i}>0$ such that

$$
\psi_{k i}\left(W_{i}(t, x(t))\right) \leq \frac{c_{k k}(\sigma)}{k+1}, \quad t \geq t_{0}+\tilde{T}_{i} .
$$

Since $e_{k}(t)$ is integrable over $R_{+}$, there is a $T^{*}>0$ such that

$$
e_{k}(t) \leq \frac{c_{k k}(\sigma)}{k+1}, \quad t \geq t_{0}+T^{*}
$$

It then follows from Eqs. (3.15)-(3.18) that for $i$ sufficiently large

$$
D^{+} W_{k}(t, x(t)) \leq \frac{c_{k k}(\sigma)}{k+1}, \quad t \in\left[\alpha_{i}, \beta_{i}\right],
$$

which implies that $W_{k}(t, x(t))$ is decreasing on $\left[\alpha_{i}, \beta_{i}\right]$ and hence $W_{k}\left(\beta_{i}, x\left(\beta_{i}\right)\right)<$ $W_{k}\left(\alpha_{i}, x\left(\alpha_{i}\right)\right)$, which is a contradiction to (3.16). Thus $\lim _{t \rightarrow \infty} W_{k}(t, x(t))=0$. It therefore follows by induction that

$$
\lim _{t \rightarrow \infty} W_{i}(t, x(t))=0, \quad i=1,2, \ldots, N .
$$

Since $W_{i}(t, x)$ is positive definite with respect to $E_{i}$ for each $i=1,2, \ldots, N$, we conclude $x(t) \rightarrow \bigcap_{n=1}^{\infty} E_{i}$ as $t \rightarrow \infty$. The proof is complete.

EXAMPLE 3.2. Consider the nonlinear system

$$
\left\{\begin{array}{l}
x_{1}^{\prime}=-a_{1} \cos ^{2} t x_{1}^{\gamma_{1}}+\frac{e_{1}(t)}{1+\sum_{i=1}^{N} x_{i}^{2}}, \\
x_{2}^{\prime}=\frac{1}{1+x_{1}^{2}} f_{21}\left(x_{1}\right)-a_{2} x_{2}^{\gamma_{2}}+\frac{e_{2}(t)}{1+\sum_{i=1}^{N} x_{i}^{2}}, \\
\ldots \ldots \\
x_{N}^{\prime}=\frac{1}{1+x_{N}^{2}}\left[\sum_{j=1}^{N-1} f_{N_{j}}\left(x_{j}\right)\right]-a_{N} x_{N}^{\gamma_{N}}+\frac{e_{N}(t)}{1+\sum_{i=1}^{N} x_{i}^{2}},
\end{array}\right.
$$


where $a_{i}>0, e_{i}(t)$ is integrable over $R_{+}, \gamma_{i}$ is a positive odd integer for each $i=$ $1,2, \ldots, N, f_{i j}\left(x_{j}\right)$ is continuous in $x_{j}$, and $f_{i j}(0)=0$ for $i=1,2,3, \ldots, N$, $j=1,2, \ldots, i-1$. Choose $V=W_{1}=x_{1}^{2}, W_{i}=x_{i}^{2}, i=1,2, \ldots, N$. Then it is not difficult to verify that all conditions of Theorem 3.2 are satisfied with $E_{i}=\left\{\left(x_{1}, \ldots, x_{N}\right) \in R^{N}, x_{i}=0\right\}$. Thus we conclude that $\left(x_{1}(t), \ldots, x_{N}(t)\right) \rightarrow$ $(0, \ldots, 0)$ as $t \rightarrow \infty$ for all bounded solutions of Eqs. (3.19).

As another application of the above results, let us split the system (2.1) as

$$
\left\{\begin{array}{l}
x_{1}^{\prime}=f_{1}\left(t, x_{1}, x_{2}\right), \\
x_{2}^{\prime}=f_{2}\left(t, x_{1}, x_{2}\right),
\end{array}\right.
$$

where $x=\operatorname{col}\left(x_{1}, x_{2}\right), f=\operatorname{col}\left(f_{1}, f_{2}\right), x_{1} \in R^{p}, x_{2} \in R^{q}$ with $p+q=n$. Then we have the following result.

Theorem 3.3. Assume that

(i) $V \in C\left[R_{+} \times \Omega, R_{+}\right], V(t, x)$ is locally Lipschitz in $x$ and positive definite, $V(t, 0) \equiv 0$, and

$$
D^{+} V(t, x) \leq-\lambda(t) C_{1}\left(\left\|x_{2}\right\|\right), \quad(t, x) \in R_{+} \times \Omega,
$$

where $\lambda(t)$ is integrally positive and $C_{1} \in K$;

(ii) $f \in C\left[R_{+} \times \Omega, R^{n}\right], f(t, 0) \equiv 0$, and there exist constants $M, L>0$ such that for $(t, x) \in R_{+} \times \Omega$,

$$
\begin{aligned}
x_{2} \cdot f_{2}(t, x) & \leq M, \\
\left\|f_{1}\left(t, x_{1}, x_{2}\right)-f_{1}\left(t, \bar{x}_{1}, \bar{x}_{2}\right)\right\| & \leq L\left(\left\|x_{1}-\bar{x}_{1}\right\|+\left\|x_{2}-\bar{x}_{2}\right\|\right) ;
\end{aligned}
$$

(iii) the trivial solution of the subsystem

$$
x_{1}^{\prime}=f_{1}\left(t, x_{1}, 0\right)
$$

is uniformly asymptotically stable.

Then the trivial solution of Eqs. (3.20) is asymptotically stable.

Proof. The main idea of the proof is to utilize the converse theorem for the subsystem (3.21) and obtain a Lyapunov function $W_{2}(t, x)$ and then prove, incorporating conditions (ii), that $W_{2}(t, x)$ satisfies condition (ii) of Theorem 3.1. We omit the details here.

EXAMPLE 3.3. Consider the nonlinear system in $R^{2}$

$$
\left\{\begin{array}{l}
x_{1}^{\prime}=-\frac{1}{2} x_{1}-\left(1+\cos ^{2} t\right) x_{2}, \\
x_{2}^{\prime}=x_{1}-2 x_{1} x_{2}^{2}-\left(5+4 \cos ^{2} t\right) x_{2}^{3} .
\end{array}\right.
$$

Let $V\left(t, x_{1}, x_{2}\right)=2 x_{1}^{2}+2 x_{1} x_{2}+\left(\frac{5}{2}+2 \cos ^{2} t\right) x_{2}^{2}$. Then computing the time derivative of $V$ along solutions of Eqs. (3.22), we obtain

$$
D^{+} V\left(t, x_{1}, x_{2}\right) \leq-2 \cos ^{2} t x_{2}^{2} .
$$

We see that $V\left(t, x_{1}, x_{2}\right)$ is positive definite and $V(t, 0,0) \equiv 0$. Clearly, $\left|f_{1}\left(t, x_{1}, x_{2}\right)-f_{1}\left(t, \bar{x}_{1}, \bar{x}_{2}\right)\right| \leq 2\left(\left|x_{1}-\bar{x}_{1}\right|+\left|x_{2}-\bar{x}_{2}\right|\right) \quad$ and $\quad f_{1}\left(t, x_{1}, x_{2}\right)=-\frac{1}{2} x_{1}$. 
Thus the trivial solution of the subsystem

$$
x_{1}^{\prime}=-\frac{1}{2} x_{1}
$$

is uniformly asymptotically stable. Hence all conditions of Theorem 3.3 are satisfied, and we conclude that the trivial solution of Eqs. (3.22) is globally asymptotically stable.

To conclude the paper, we emphasize that our method is essentially an extension of the key ideas in the invariance principle to nonautonomous systems, which also shows that in those cases where the Lyapunov function found does not satisfy the desired conditions, it is fruitful to utilize it as a basis for further refinement than to discard it. On the other hand, this approach can also be applied to autonomous systems in cases where a single attractive set and the invariance properties of the limit sets fail to yield the desired result. From this point of view, our method may also be considered as a further refinement of LaSalle's invariance principle.

\section{REFERENCES}

[1] E. A. Barbashin and N. N. Krasovskii, On global stability of motion, Dokl. Akad. Nauk SSSR 86, 453-456 (1952)

[2] T. A. Burton, An extension of Liapunov's direct method, J. Math. Anal. Appl. 28, 545-552 (1960); 32, 689-691 (1970)

[3] J. Haddock, Stability theory for nonautonomous systems, Dynamical Systems, Proc. International Symposium, Vol. II, Brown Univ., Providence, RI, 1974, L. Cesari, J. Hale, and J. LaSalle, eds., Academic Press, New York, 1976, pp. 599-603

[4] L. Hatvani, Attractivity theorems for non-autonomous systems of differential equations, Acta Sci. Math. 40, 271-283 (1978)

[5] L. Hatvani, On the uniform attractivity of solutions of ordinary differential equations by two Lyapunov functions, preprint, 1990

[6] V. Lakshmikanthan and Xinzhi Liu, On asymptotic stability for non-autonomous differential systems, Nonlinear Anal. 13, 1181-1189 (1989)

[7] J. P. LaSalle, The stability of dynamical systems, Regional Conference Series in Applied Mathematics, SIAM, Philadelphia, PA, 1976

[8] J. P. LaSalle, Stability of nonautonomous systems, Nonlinear Anal. 1, 83-91 (1976)

[9] Xinzhi Liu, Perturbing families of Lyapunov functions and asymptotic stability, J. Math. Phys. Sci. 23, 23-34 (1989)

[10] V. M. Matrosov, On the theory of stability of motion, Prikl. Mat. Mekh. 26, 992-1002 (1962)

[11] L. Salvadori, Some contributions to asymptotic stability theory, Ann. Soc. Sic. Bruxelles Sér. I 88, 183-194 (1974)

[12] T. Yoshizawa, Asymptotic behavior of solutions of a system of differential equations, Contrib. Differential Equations 1, 371-387 (1963)

[13] T. Yoshizawa, Attractivity in non-autonomous systems, Internat. J. Non-linear Mech. 20, 519-528 (1985) 